\title{
Differential colorimetry measurements of fluctuation growth in nanofilms exposed to large surface thermal gradients
}

Cite as: J. Appl. Phys. 125, 065303 (2019); https://doi.org/10.1063/1.5051456

Submitted: 08 August 2018 . Accepted: 24 January 2019. Published Online: 12 February 2019

Kevin R. Fiedler (D), Euan McLeod, and Sandra M. Troian
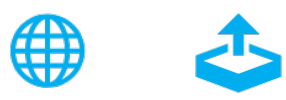

View Online

Export Citation

\section{ARTICLES YOU MAY BE INTERESTED IN}

Hollow cathode effect modified time-dependent global model and high-power impulse magnetron sputtering discharge and transport in cylindrical cathode

Journal of Applied Physics 125, 063302 (2019); https://doi.org/10.1063/1.5048554

Magnetoresistance of a single polycrystalline nickel nanowire

Journal of Applied Physics 125, 063902 (2019); https://doi.org/10.1063/1.5064680

Temperature variation of the isothermal bulk modulus in solids: Thermo-elastic instability and melting

Journal of Applied Physics 125, 065104 (2019); https://doi.org/10.1063/1.5078722

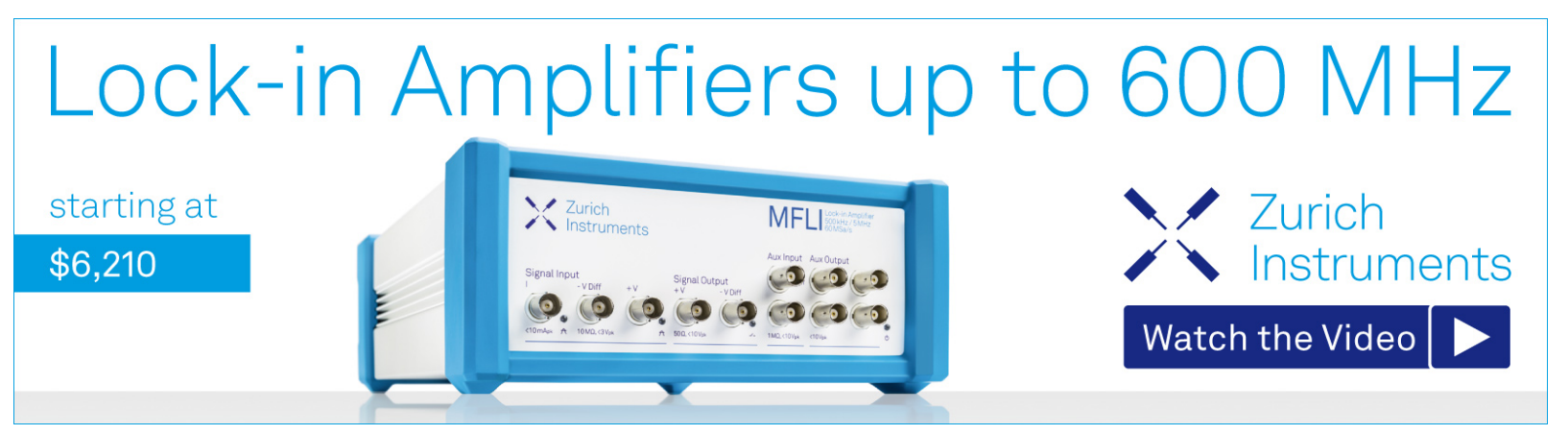




\title{
Differential colorimetry measurements of fluctuation growth in nanofilms exposed to large surface thermal gradients
}

\author{
Cite as: J. Appl. Phys. 125, 065303 (2019); doi: 10.1063/1.5051456 \\ Submitted: 8 August 2018 - Accepted: 24 January 2019 . \\ Published Online: 12 February 2019
}

Kevin R. Fiedler, ${ }^{1, a)}$ (D) Euan McLeod, ${ }^{2}$ and Sandra M. Troian ${ }^{7, b)}$

\author{
AFFILIATIONS \\ ${ }^{1}$ California Institute of Technology, T. J. Watson Sr. Laboratories of Applied Physics, MC 128-95, Pasadena, California 91125, USA \\ ${ }^{2}$ College of Optical Sciences, University of Arizona, 1630 E. University Blvd, P.O. Box 210094, Tucson, Arizona 85721, USA \\ a) Present address: Department of Mathematics and Statistics, Washington State University, 2710 Crimson Way, Richland, \\ Washington 99354, USA.

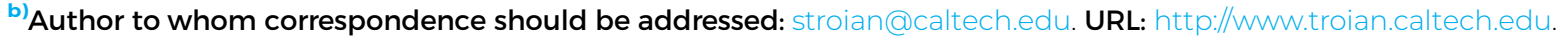

\begin{abstract}
Slender liquid nanofilms exposed to large surface thermal gradients are known to undergo thickness fluctuations, which rapidly self-organize into arrays of nanoprotrusions with a separation distance of tens of microns. We previously reported good agreement between measurements of the characteristic spacing and the wavelength of the most unstable mode predicted by a linear stability analysis based on a long wavelength thermocapillary model. Here, we focus on differential colorimetry measurements to quantify early time out-of-plane growth of protrusions for peak heights spanning 20 to $200 \mathrm{~nm}$. Analysis of peak heights based on shape reconstruction reveals robust exponential growth. Good quantitative agreement of the growth rates with the thermocapillary model is obtained using a single fit constant to account for material parameters of nanofilms that could not be measured directly. These findings lend further support to the conjecture that the array protrusions uncovered almost two decades ago likely stem from a linear instability, whose growth rate is controlled by thermocapillary forces counterbalanced by capillary forces.
\end{abstract}

Published under license by AIP Publishing. https://doi.org/10.1063/1.5051456

\section{BACKGROUND}

Experiments designed to elicit the physical mechanisms generating linear instabilities in macroscale fluid systems normally rely on early time measurements of an emergent length or time scale signalling the growth of the most unstable wavelength. This is common to measurements in many large scale systems, which manifest stationary periodic, oscillatory uniform, or oscillatory periodic phenomena. At the macroscale, such measurements can often be obtained by direct visualization. With growing interest in small scale fluidic phenomena, similar measurements pose more challenges-not only do films easily rupture or are otherwise compromised by defects but measurements must often rely on indirect techniques from which key parameter values are inferred. At microscale or nanoscale dimensions, matching system size and material properties to the appropriate measurement technique often severely restricts the options available. For films whose thickness is of the order of tens of microns or more, laser or white light interferometry, sometimes coupled with shadowgraphy, is often the tool of choice. ${ }^{1-3}$

For more than a decade now, there has been interest in identifying the source of various runaway instabilities in even thinner films, which undergo spontaneous transition from an initially flat and uniform layer to microarrays containing nanoprotrusions. In these systems, fluid elongations tend to grow without bound unless prematurely terminated by direct contact with an opposing substrate or by fluid depletion effects. Early time measurements of the fastest growing wavelength in polymeric nanofilms subject either to large surface electric field gradients ${ }^{4-7}$ or large surface thermal 
gradients $^{8-11}$ have yielded a characteristic in-plane separation distance of the order of tens of microns. Current understanding of these systems is that capillary forces, which suppress development of regions of high interfacial curvature, are counterbalanced and then eventually dominated either by electrohydrodynamic or by thermocapillary forces which rapidly undergo self-reinforcement, leading to fluid elongations with long range order.

Experimentalists investigating the electrohydrodynamic instability have had some success in measuring out-of-plane growth for film thicknesses of about $5 \mu \mathrm{m}$ or less. Leach et al. ${ }^{7}$ and Russell and Bae ${ }^{12}$ used laser scanning confocal microscopy with single wavelength illumination $(458 \mathrm{~nm})$ to obtain real time measurements of the film deformation process. The field of view spanned roughly $750 \mu \mathrm{m} \times 750 \mu \mathrm{m}$ and encompassed about 10 to 20 liquid peaks. Frame by frame analysis of the fringe spacing associated with the first peak to touch the counter-electrode allowed reconstruction of the evolving shapes, which seemed well described by a Gaussian function. The measured growth in peak heights was found to be consistent with exponential growth as predicted by a linear stability analysis, 6,13 although the number of data points was rather small and the scatter was significant. These measurements revealed how the growth rate was strongly influenced by the applied voltage difference, the initial film thickness, and the liquid viscosity. In a separate study, Leach et al. also used scanning electron microscopy to capture still images of film deformation which accompanied hierarchical formation in trilayer systems consisting of two polymeric films (total thickness of about $500 \mathrm{~nm}$ ) overlaid by an air layer. ${ }^{14}$

In this work, we focus on early time measurements of the out-of-plane growth of nanoprotrusions triggered by thermal gradients in much thinner films. This initial period of growth poses significant experimental challenges since emergent fluctuations in film thickness measure less than a few tens of nanometers. We therefore resort to a technique based on differential colorimetry applied to surface reflectivity images of ultrathin transparent films. While the method in principle is well suited to measurement of liquid peak growth in nanoscale films, the material films themselves pose problems because they are prone to defects from dust and other contaminants. These defects, which can be embedded or exposed, tend to trigger localized nucleation and growth, which interfere with measurements of the native instability. Gathering statistically relevant data from a sufficiently large region of a film not compromised by such defects proves a challenging task.

In what follows, we report the first measurements of the early time growth rate of liquid protrusions spanning 20 to $200 \mathrm{~nm}$ in height in nanofilms exposed to large surface thermal gradients. These measurements rely on frame by frame tracking of the ten most rapidly growing liquid peaks which are monitored and analyzed using differential colorimetry. ${ }^{15}$ The experimental design allowed a fairly large field of view (about $1.36 \mathrm{~mm} \times 1.08 \mathrm{~mm}$ ) which contained about 100 liquid peaks per frame. Reconstruction of peak heights at early times revealed robust exponential growth. The measured growth rates were found to compare well quantitatively to predictions of a long wavelength thermocapillary model ${ }^{8-11}$ using a single adjustable parameter to account for material parameters of nanofilms that could not be measured directly.

\section{INSTABILITY GROWTH RATE ESTIMATE FROM THE LONG WAVELENGTH THERMOCAPILLARY MODEL}

Dietzel and Troian ${ }^{8,9}$ previously conducted a linear stability analysis of the interface equation governing the evolution of the local film thickness $h(\vec{x}, t)<d_{0}$, where $\vec{x}=(x, y)$ denotes the in-plane coordinates (and $z$ is the out-of-plane coordinate). According to this model, an initially flat, uniform liquid nanofilm of thickness $h_{0}$ sandwiched between two solid substrates separated by a very small distance $d_{0}$ and maintained at a uniform temperature difference $\Delta \mathrm{T}=\mathrm{T}_{\mathrm{H}}-\mathrm{T}_{\mathrm{C}}$, where $\mathrm{H}$ and $\mathrm{C}$ designate hot and cold, respectively, will spontaneously undergo deformation to a periodic array of liquid protrusions characterized by an in-plane spacing $\lambda_{0}$, which in the experiment typically spans several tens of microns. The quantity $\lambda_{0}$ denotes the wavelength of the fastest growing unstable mode. The slender gap width $d_{0}$, which is typically less than a couple microns in the experiment, gives rise to an extremely large nominal temperature gradient $\Delta \mathrm{T} / d_{0}$ responsible for triggering a thermocapillary instability. Depicted in Fig. 1 is a sketch representing a cross-sectional view of a linearly unstable nanofilm with peak thickness $\left.h_{\text {peak }} \propto \exp \left[\sigma\left(k_{0}\right) t\right)\right]$, where $k_{0}=2 \pi \lambda_{0}$.

This model incorporates various assumptions and approximations. The liquid nanofilm is assumed to be a Newtonian liquid overlaid by a slender quiescent, inviscid gas layer. The film thickness must satisfy $0<h(\vec{x}, t)<d_{0}$ which precludes film rupture from occurring along the bottom hot substrate or liquid contact with the upper cold substrate. The slender gap approximation (sometimes called the thin film lubrication approximation) given by $\left(h_{0} / \lambda_{0}\right)^{2}<\left(d_{0} / \lambda_{0}\right)^{2} \ll 1$ greatly simplifies the form of the Navier-Stokes equation and thermal

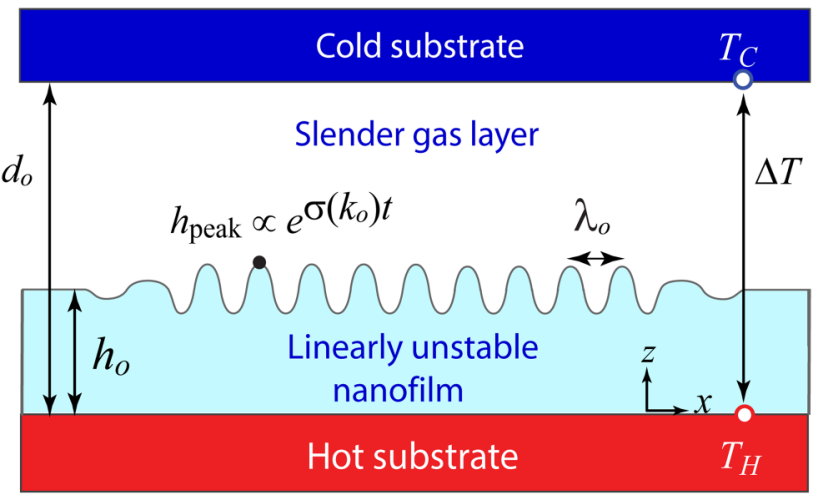

FIG. 1. Sketch of an unstable liquid nanofilm with initial thickness $h_{0}$ sandwiched between two substrates separated by a small distance $d_{0}$ and maintained at a uniform temperature difference $\Delta T=T_{H}-T_{C}$, where $H$ and $C$ designate hot and cold, respectively. The fastest growing unstable mode with wave number $k_{0}=2 \pi / \lambda_{0}$ is characterized by liquid peak amplitudes $h_{\text {peak }} \propto e^{\sigma\left(k_{0}\right) t}$. The dispersion relation for $\sigma\left(k_{0}\right)$ is given by Eq. (3). 
equation used to derive the interface equation. The material parameters characterizing the nanofilm, namely, the viscosity $\eta$, surface tension $\gamma$, thermocapillary coefficient $d \gamma / d \mathrm{~T}<0$, and thermal conductivity ratio $\kappa=k_{\text {gas }} / k_{\text {liquid, }}$, are evaluated at the temperature of the liquid/solid substrate and assumed to be constant at early times when fluctuations in thickness or interfacial temperature are infinitesimally small, as predicated by linear stability theory.

Within the long wavelength approximation, the dominant thermal gradient is given by $\partial \mathrm{T}(z, t) / \partial z$, which is established by thermal conduction. Estimates of the magnitude of thermal convection and radiation indicate that those heat fluxes are smaller than the thermal conductive flux by several orders of magnitude. , $^{9,1}$ As a result, the majority of the vertical temperature drop in the slender gap occurs within the gas layer due its low thermal conductivity in comparison to the liquid film. Additionally, it is assumed that no phase change occurs during the instability, which is ensured as long as the temperature of the colder substrate is maintained above the melting point of the liquid nanofilm.

The governing interface equation for the evolving film thickness $h(x, y, t)$ is then given by

$$
\frac{\partial h}{\partial t}+\nabla_{\|} \cdot\left\{\frac{\gamma h^{3}}{3 \eta} \nabla_{\|} \nabla_{\|}^{2} h+\frac{\kappa|d \gamma / d \mathrm{~T}| \Delta \mathrm{T} d_{o} h^{2} \nabla_{\|} h}{2 \eta\left[d_{o}+h(\kappa-1)\right]^{2}}\right\}=0
$$

where $\nabla_{\|}=(\partial / \partial x, \partial / \partial y)$. The first term in brackets quantifies the leveling action of capillary forces, while the second term describes the shearing action of thermocapillary forces which promote the growth of ever taller peaks. The interested reader is referred to Ref. 9 for the complete derivation leading to Eq. (1) and the dispersion relation described next.

According to this model, small disturbances in film thickness or interfacial temperature give rise to large thermocapillary stresses at the gas/liquid interface mitigated only by capillary stresses since gravitational forces are negligible at these small length scales. A modal stability analysis reveals that at early times, an initially flat liquid film will therefore become linearly unstable to periodic disturbances and undergo exponentially rapid and spontaneous deformation described by

$$
h(\vec{x}, t)=h_{0}+\delta h e^{\sigma(k) t} e^{i \vec{k} \cdot \vec{x}}
$$

where $\delta h \ll h_{0}$ and $k=|\vec{k}(x, y)|=2 \pi / \lambda$. A positive growth rate $\sigma(k)$ (which is purely real) is indicative of instability, while negative values indicate modal decay reverting back to the initial state $h_{0}$. The general dispersion curve obeys a Type II instability ${ }^{16}$ of the form

$$
\sigma(k)=\frac{h_{o} k^{2}}{\eta}\left[\frac{\kappa|d \gamma / d T| D \Delta T}{2(D+\kappa-1)^{2}}-\frac{\gamma h_{o}^{2}}{3} k^{2}\right]
$$

where $D=d_{o} / h_{o}$ is the normalized substrate separation distance.
The extremum of Eq. (3) yields an expression for the wavenumber of the fastest growing unstable mode $k_{0}=$ $\left|\vec{k}_{0}(x, y)\right|$ given by

$$
k_{0}=\frac{2 \pi}{\lambda_{0}}=\frac{1}{h_{0}} \sqrt{\frac{3 \kappa|d \gamma / d T| \Delta T}{4 \gamma}} \frac{\sqrt{\mathrm{D}}}{(\mathrm{D}+\kappa-1)},
$$

whose growth rate $\sigma_{0}$ is given by the relation

$$
\sigma_{o}=\frac{3 \kappa^{2}|d \gamma / d T|^{2}(\Delta T)^{2}}{16 \eta \gamma h_{o}} \frac{D^{2}}{(D+\kappa-1)^{4}}
$$

For later use, we also here introduce the relation between the non-dimensional maximal growth rate $\Sigma_{0}$ and the normalized wavelength $\Lambda_{0}=\lambda_{0} / h_{0}$ for the fastest growing mode, namely,

$$
\Sigma_{0}=\frac{\eta h_{o}}{\gamma} \sigma_{o}=\frac{1}{3}\left(\frac{2 \pi}{\Lambda_{0}}\right)^{4}
$$

This relation holds for any Type-II instability, where the dispersion curve $\sigma(k)$ consists of a positive quadratic term dominant at small wave numbers $k$ counteracted by a negative quartic term dominant at large wave numbers. For the long wavelength instability of interest, the quadratic term represents the destabilizing thermocapillary forces and the quartic term represents stabilizing capillary forces.

\section{BRIEF REVIEW OF THE EXPERIMENTAL SYSTEM}

Here, we briefly review key elements of the sample preparation techniques and assembly of the experimental setup depicted in Fig. 2. The interested reader may wish to consult prior work ${ }^{10,11}$ where additional details are provided, including description of the finite element simulations conducted for estimating $\Delta \mathrm{T}$.

The molten nanofilms used in this study were made from a polystyrene standard $(1.3 \mathrm{~kg} / \mathrm{mol} \mathrm{PS}$, polydispersity index $\mathrm{M}_{w} / \mathrm{M}_{n}=1.10$, Scientific Polymer Products, Inc.), which was dissolved in toluene then filtered to remove any undissolved or agglomerated material using a $0.2 \mu \mathrm{m}$ PTFE filter (Cole Palmer) with $200 \mathrm{~nm}$ nominal pore size. The solutions were then spun coat onto the polished side of a silicon wafer to the desired thickness (CEE-100, Brewer Science). The coated wafers were then vacuum baked at $80^{\circ} \mathrm{C}$ for $2 \mathrm{~h}$ to remove residual solvent (Precision Model 19, Thermo Fisher Scientific). The thickness of the resulting solid film, measured by ellipsometry (Gaertner Model L166C), ranged from about 95 to $390 \mathrm{~nm}$. Film thickness measurements were obtained by averaging values at three locations of the film, which yielded variation of no more than $5 \mathrm{~nm}$. The average values of film thickness $h_{0}$ are listed in Table I.

The top cooled substrate depicted in Fig. 2 consisted of a cylindrical sapphire window (Edmund Optics, single crystal, diameter $7.5 \mathrm{~mm}$, thickness $400 \pm 50 \mu \mathrm{m}$ ) chosen for its high optical transparency and high thermal conductivity. 


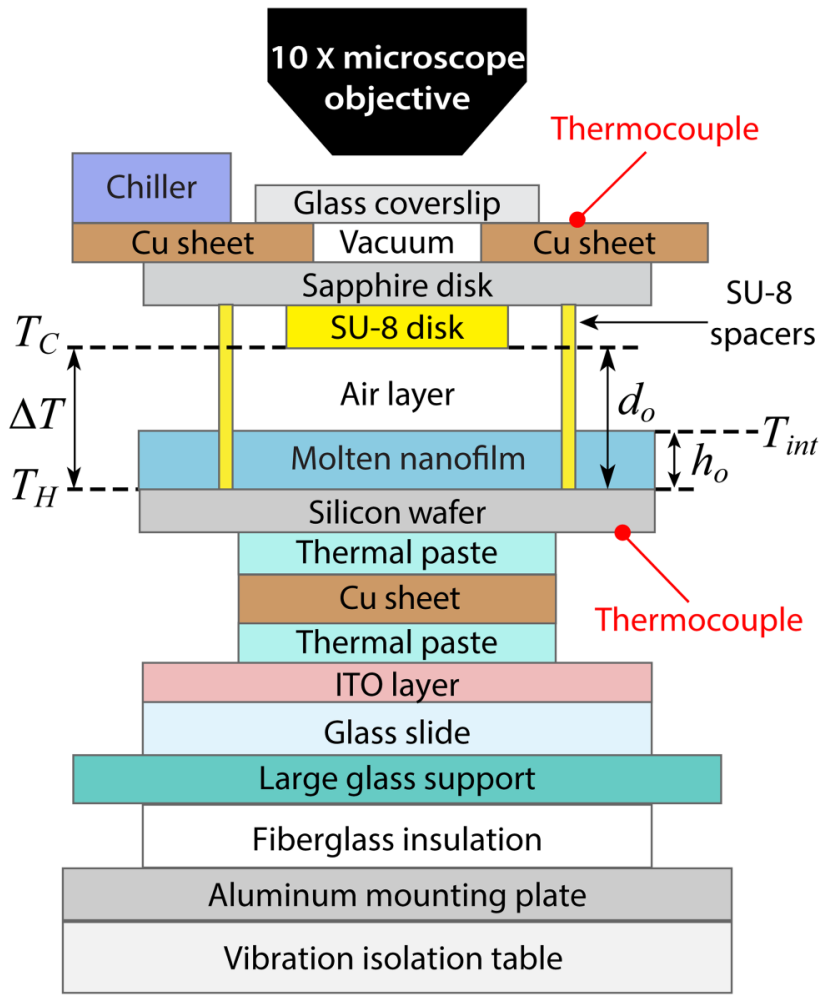

FIG. 2. Diagram of the geometry and material layers comprising the experimental system (not drawn to scale). Exact geometric dimensions, material constants, and other relevant information for each layer can be found in Ref. 11. Relevant ranges of experimental values can be found in Table II and Table III.

The sapphire was patterned with four optically transparent cylindrical posts made of photoresist (MicroChem, SU-8 2000). These posts, which were measured to be approximately $2.2 \mu \mathrm{m}$ in length and $0.010 \mathrm{~mm}^{2}$ in area, were lithographically patterned at the vertices of a square measuring $3.0 \mathrm{~mm}$ on the diagonal. These posts were used as gap spacers to help maintain a fixed distance between the hot and cold substrates. Centered between these spacers was a wide and slender cylindrical transparent disk (also fabricated of MicroChem, SU-8 2000) measuring approximately $1.0 \mathrm{~mm}$ in diameter and either $1.38 \mu \mathrm{m}$ or $1.48 \mu \mathrm{m}$ in thickness. Experiments numbered 66 and 69 in Table I were conducted with a bare sapphire window containing no SU-8 disk. The thicknesses of the disk and posts were measured with a stylus profilometer (Ambios XP-2). The separation distances $d_{0}$ listed in Table I were obtained by averaging the measured lengths of the four supporting posts and then subtracting the value of the disk thickness measured at its center. When no disk was used, the value $d_{0}$ equaled the average spacer distance. The variation in measured post lengths on a single disk never exceeded about $7 \%$ and was typically closer to $2-3 \%$.
This assembly was positioned under a microscope using a translational stage (Prior, Model Type HT1111). Perfect parallelism between the sapphire window and $\mathrm{Si}$ wafer was difficult to achieve over an extended region due to intrinsic limitations in substrate flatness (Sapphire, $2 \lambda @ 632.8 \mathrm{~nm}$ ) and variation in wafer thickness ( $\mathrm{Si}$ wafer, about $5 \mu \mathrm{m}$ ) and challenges inherent with manual leveling which relied on three long vertical screws used to attach the bottom aluminum mounting plate to the top $\mathrm{Cu}$ sheet. The leveling process was carried out visually by adjusting the screws so as to minimize interference fringes caused by the air layer above the coated wafer to a level about $100 \mathrm{~nm} / \mathrm{mm}$. It was often the case, however, that the substrates had to be leveled again after the indium tin oxide (ITO) heater was turned on due to thermal expansion within the optical path. These corrections were performed within the first five minutes or so of commencing a run, after which the system appeared to remain fairly stable.

To begin a run, the chiller was turned on and the PS coated wafer was positioned as shown in Fig. 2. The chiller was used to cool the sapphire disk and SU-8 disk to temperatures well above the PS glass transition temperature so that the nanofilm always remained in a molten viscous state. Power was then applied to the indium tin oxide (ITO) coated glass slide to initiate heating and melting of the PS film. Our measurements indicated a glass transition temperature of about $\mathrm{T}_{g}=60^{\circ} \mathrm{C}$ based on observational measurements of the transition to a viscous liquid, consistent with reported values. ${ }^{18}$ Once the temperature of the heated wafer reached steady state, which normally required from 1.5 to $5 \mathrm{~min}$, data collection began. All the images analyzed in this study (e.g., Fig. 6) were obtained well after the temperature had stabilized so that the temperatures $\mathrm{T}_{\mathrm{H}}$ and $\mathrm{T}_{\mathrm{C}}$ remained constant during the course of measurement.

Digital images showing the growth of liquid protrusions were obtained with a color CCD camera (DVC 1312C) mounted onto the porthole of a Zeiss Axiovert 200 MAT microscope at $10 \times$ magnification. A halogen light (100 W max., Osram, HLX $64625)$ provided the free space illumination. Images were captured every one to two minutes by a frame grabber card (PIXCI-D) and image capture software (XCAP, Epix Inc.). All the interstitial layers situated between the silicon wafer and glass coverslip, including the SU-8 photoresist disk (when in place), were optically transparent to the halogen light.

In order to obtain estimates of the instability growth rate, values of the temperature difference $\Delta T=T_{H}-T_{C}$ were required as inputs to Eq. (5). Direct measurement of the values of $\Delta \mathrm{T}$ was not possible since the separation distance $d_{o}$ (605 to $2200 \mathrm{~nm}$ ) was too small to accommodate even the smallest of thermocouples available commercially. Infrared imaging was also not used since it lacked the resolution needed to acquire reliable estimates of $\Delta \mathrm{T}(\vec{x}, t)$. We initially also conducted a review of the literature to determine how the quantity $\Delta \mathrm{T}(\vec{x}, \mathrm{t})$ was obtained in prior experiments conducted by other researchers. ${ }^{4,5,20-22}$ Our review revealed that the values so far reported were actually the set point values for the hot and cold stages and not the actual temperature drops across the air/liquid bilayer, indicating that the literature values 
TABLE I. Parameter values for all experiments examined in this study. Dimensions and symbols refer to the system geometry depicted in Fig. 2-additional layer thicknesses are listed in Table III. Columns below denote the following: Expt. is the number assigned each run using the same numbering convention as used in Ref. 11; $d_{0}$ is the separation distance between the proximal hot and cold substrates confining the liquid film; $h_{0}=$ is the initial thickness of the PS film; $D=d_{0} / h_{0}$ is the normalized separation distance; SU-8 denotes the thickness of the SU-8 disk; $\lambda_{0}$ is the wavelength of the fastest growing unstable mode, previously measured and reported in Ref. 11; $T_{H}$ and $T_{C}$ denotes the temperatures of the (hot) PS/silicon interface and the (cold) air/SU-8 interface [or the (cold) air/sapphire interface when no SU-8 disk was present) as computed from finite element simulations previously reported in Ref. 11; $\Delta T=T_{H}-T_{C} ; \eta\left(T_{H}\right)$ and $\eta\left(T_{C}\right)$ denote the PS viscosity values extrapolated from Fig. 7 of Ref. 18 ; $\sigma_{0}^{\min }, \sigma_{0}^{\max }$, and $\sigma_{0}^{\text {avg }}$ denote the minimum, maximum, and average values of the measured growth rates for the ten fastest growing peaks listed in Table $V$.

\begin{tabular}{|c|c|c|c|c|c|c|c|c|c|c|c|c|c|c|}
\hline Expt. & $\begin{array}{c}d_{o} \\
(\mathrm{~nm})\end{array}$ & $\begin{array}{c}h_{\circ} \\
(\mathrm{nm})\end{array}$ & $D$ & $\begin{array}{l}\text { SU-8 } \\
(\mu \mathrm{m})\end{array}$ & $\begin{array}{c}\lambda_{0} \\
(\mu \mathrm{m})\end{array}$ & $\begin{array}{c}T_{H} \\
\left({ }^{\circ} \mathrm{C}\right) \\
\end{array}$ & $\begin{array}{l}T_{\text {Int }} \\
\left({ }^{\circ} \mathrm{C}\right)\end{array}$ & $\begin{array}{c}T_{C} \\
\left({ }^{\circ} \mathrm{C}\right) \\
\end{array}$ & $\begin{array}{c}\Delta T \\
\left({ }^{\circ} \mathrm{C}\right)\end{array}$ & $\begin{array}{l}\eta\left(T_{H}\right) \\
(\mathrm{Pas}) \\
\end{array}$ & $\begin{array}{l}\eta\left(T_{C}\right) \\
(\mathrm{Pa} \mathrm{s}) \\
\end{array}$ & $\begin{array}{c}\sigma_{o}^{\min } \\
\left(10^{-4} / \mathrm{s}\right)\end{array}$ & $\begin{array}{c}\sigma_{0}^{\max } \\
\left(10^{-4} / \mathrm{s}\right) \\
\end{array}$ & $\begin{array}{c}\sigma_{\circ}^{\text {avg }} \\
\left(10^{-4} / \mathrm{s}\right) \\
\end{array}$ \\
\hline 46 & 900 & 189 & 4.76 & 1.38 & 37.0 & 93.6 & 92.4 & 73.8 & 19.8 & 60.6 & 925 & 4.11 & 5.89 & 5.03 \\
\hline 47 & 810 & 189 & 4.29 & 1.38 & 40.9 & 99.0 & 98.1 & 86.3 & 12.7 & 36.1 & 152 & 2.94 & 7.79 & 4.18 \\
\hline 48 & 840 & 183 & 4.59 & 1.38 & 32.5 & 89.9 & 88.6 & 69.3 & 20.6 & 93.9 & 2440 & 3.84 & 5.68 & 4.78 \\
\hline 50 & 900 & 181 & 4.97 & 1.38 & 48.5 & 96.8 & 95.8 & 80.8 & 16.0 & 45.2 & 323 & 4.99 & 8.13 & 6.58 \\
\hline 51 & 700 & 181 & 3.87 & 1.48 & 38.6 & 91.8 & 90.5 & 75.4 & 16.4 & 76.5 & 755 & 2.70 & 8.74 & 7.01 \\
\hline 52 & 605 & 185 & 3.27 & 1.48 & 39.1 & 98.5 & 97.6 & 88.7 & 9.83 & 37.2 & 105 & 9.45 & 17.9 & 13.1 \\
\hline 53 & 830 & 183 & 4.73 & 1.38 & 59.1 & 101 & 99.7 & 88.1 & 12.4 & 33.0 & 116 & 2.87 & 5.23 & 3.79 \\
\hline 54 & 670 & 185 & 3.62 & 1.48 & 35.6 & 99.0 & 98.1 & 88.4 & 10.6 & 36.2 & 109 & 4.73 & 10.7 & 7.54 \\
\hline 56 & 885 & 183 & 4.84 & 1.38 & 37.2 & 89.8 & 88.6 & 67.8 & 22.1 & 94.4 & 3930 & 2.39 & 4.68 & 3.50 \\
\hline 58 & 760 & 142 & 5.35 & 1.48 & 37.6 & 96.1 & 95.3 & 81.1 & 15.0 & 48.3 & 310 & 1.83 & 5.51 & 3.60 \\
\hline 60 & 735 & 95.0 & 7.74 & 1.48 & 29.1 & 95.9 & 95.4 & 80.4 & 15.5 & 49.3 & 337 & 2.91 & 5.65 & 3.79 \\
\hline 61 & 710 & 101 & 7.03 & 1.48 & 42.0 & 95.8 & 95.2 & 80.9 & 14.9 & 50.0 & 319 & 2.16 & 6.10 & 4.41 \\
\hline 62 & 785 & 258 & 3.04 & 1.48 & 33.4 & 93.9 & 92.2 & 78.2 & 15.7 & 59.3 & 461 & 7.03 & 12.1 & 9.84 \\
\hline 63 & 754 & 201 & 3.75 & 1.48 & 42.9 & 94.1 & 92.9 & 78.7 & 15.5 & 58.0 & 413 & 5.09 & 12.8 & 8.65 \\
\hline 64 & 615 & 215 & 2.86 & 1.48 & 39.0 & 93.1 & 91.6 & 80.1 & 13.0 & 64.6 & 351 & 10.4 & 23.8 & 15.1 \\
\hline 66 & 2200 & 156 & 14.1 & 0.00 & 66.8 & 101 & 101 & 81.0 & 20.3 & 30.9 & 314 & 0.345 & 1.38 & 0.973 \\
\hline 69 & 2200 & 184 & 12.0 & 0.00 & 41.6 & 97.2 & 96.6 & 71.4 & 25.8 & 43.2 & 1740 & 1.62 & 2.49 & 1.99 \\
\hline 71 & 850 & 366 & 2.32 & 1.48 & 53.8 & 93.7 & 91.3 & 77.4 & 16.4 & 59.9 & 549 & 12.1 & 22.5 & 17.7 \\
\hline 72 & 680 & 331 & 2.05 & 1.48 & 44.8 & 93.0 & 90.6 & 79.9 & 13.1 & 65.6 & 359 & 10.9 & 15.9 & 13.5 \\
\hline 74 & 770 & 390 & 1.97 & 1.48 & 73.2 & 96.2 & 94.0 & 84.7 & 11.5 & 47.9 & 187 & 84.5 & 158 & 122 \\
\hline
\end{tabular}

TABLE II. Dimensions and material constants for the experiments reported in this study. Nanofilms used were polystyrene melts $(1.3 \mathrm{~kg} / \mathrm{mol}$ PS standard, $M_{w} / M_{n}=1.10$, Scientific Polymer Products, Inc.). Values of the surface tension were estimated from the literature by extrapolation in molecular weight and temperature. ${ }^{17}$ Viscosities were inferred by extrapolation in temperature from the zero shear measurements reported by Urakawa et al. ${ }^{18}$ for $1.8 \mathrm{~kg} / \mathrm{mol} P S$, the molecular weight closest to our samples. Additional information regarding the values listed below can be found in Refs. 10 and 11.

\begin{tabular}{|c|c|c|}
\hline Parameter & Symbol & Value \\
\hline Initial film thickness (nm) & $h_{0}$ & $95-390$ \\
\hline Substrate separation distance $(\mathrm{nm})$ & $d_{0}$ & $605-2200$ \\
\hline Normalized separation distance $d_{0}^{\prime} / h_{0}$ & $D$ & $1.97-14.1$ \\
\hline Liquid/solid interface temp. $\left({ }^{\circ} \mathrm{C}\right)$ & $T_{H}$ & $89.8-101$ \\
\hline Air/solid interface temp. $\left({ }^{\circ} \mathrm{C}\right)$ & $T_{C}$ & $67.8-88.7$ \\
\hline Temperature difference $T_{H}-T_{C}\left({ }^{\circ} \mathrm{C}\right)$ & $\Delta T$ & $9.83-25.8$ \\
\hline $\begin{array}{l}\text { Liquid surface tension }\left(100^{\circ} \mathrm{C}\right) \\
(\mathrm{mN} / \mathrm{m}) \text { (est. from Ref. } 17)\end{array}$ & $\gamma$ & 35.5 \\
\hline $\begin{array}{l}\text { Thermocapillary coeff. }\left(\mu \mathrm{N} / \mathrm{m}-{ }^{\circ} \mathrm{C}\right) \\
\text { (est. from Ref. } 17)\end{array}$ & $|d \gamma / d T|$ & 78 \\
\hline $\begin{array}{l}\text { Thermal conductivity ratio } k_{\text {air }} / k_{\text {liquid }} \\
\text { (est. from Refs. } 17 \text { and 19) }\end{array}$ & $\kappa$ & 0.2422 \\
\hline $\begin{array}{l}\text { PS viscosity at } T_{H}(\mathrm{~Pa}-\mathrm{s}) \\
\text { (est. from Ref. 18) }\end{array}$ & $\eta\left(T_{H}\right)$ & $30.0-93.9$ \\
\hline $\begin{array}{l}\text { PS viscosity at } T_{C}(\mathrm{~Pa}-\mathrm{s}) \\
\text { (est. from Ref. 18) }\end{array}$ & $\eta\left(T_{C}\right)$ & $105-3930$ \\
\hline $\begin{array}{l}\text { Fastest growing wavelength }(\mu \mathrm{m}) \\
\text { (measured from expt.) }\end{array}$ & $\lambda_{0}$ & $29.1-73.2$ \\
\hline $\begin{array}{l}\text { Instability growth rate }\left(10^{-4} / \mathrm{s}\right) \\
\text { (measured from expt.) }\end{array}$ & $\sigma_{0}$ & $0.973-122$ \\
\hline
\end{tabular}

represent overestimates of $\Delta \mathrm{T}$. In our own experiments, we confirmed that measurements based on the set points obtained from the two thermocouple positions shown in Fig. 2 yielded large overestimates of $\Delta$ T. Such overestimates are due to the fact that the interstitial layers situated between the thermal source (ITO coated glass slide) and sink (chiller), however, thin, cause non-negligible thermal series resistances, which ultimately degrade the temperature drop across the air/liquid bilayer. Furthermore, the many edges of these interstitial layers act like thermal fins which induce further heat loss. As described in more detail in Ref. 11, our estimates of the relevant internal temperatures $T_{H}$ and $T_{C}$ were extracted from numerical simulations calibrated against actual thermocouple readings positioned exterior to the gap. The simulated values reported in Table I were substituted into Eq. (5) to obtain the predicted estimates of instability growth rates that were compared to experimental measurements.

The chromatic analysis described in Sec. IV was used to investigate 20 of the 26 experiments originally reported in Ref. 11. The same numbering convention originally used to tag experimental runs was used here as well for consistency. For various reasons, the six discarded runs did not undergo sufficient growth to be analyzed reliably by the protocol used. Relevant variables for the 20 acceptable experimental runs are provided in Tables I-III.

The remainder of this work is organized as follows. In Sec. IV, we describe the procedures and algorithms based on 
TABLE III. Layer thicknesses for glass coverslip, sapphire window, and silicon wafer depicted in Fig. 2.

\begin{tabular}{lc}
\hline \hline Layer material & Thickness $(\mathrm{mm})$ \\
\hline Corning 1737 glass coverslip & 0.150 \\
Cylindrical sapphire window & 0.400 \\
Silicon wafer & 0.675 \\
\hline \hline
\end{tabular}

differential colorimetry used to quantify the growth of liquid peak heights of the fastest growing unstable modes. In Sec. $\mathrm{V}$, we compare results of these measurements to predictions of the growth rates obtained from a linear instability analysis of a long wavelength thermocapillary model and find good agreement using a single fit parameter. In Sec. VI, we discuss limitations of this current study and suggest experimental improvements anticipated to yield even closer agreement with theory.

\section{DIFFERENTIAL COLORIMETRY MEASUREMENTS OF INSTABILITY PEAK GROWTH RATES}

\section{A. Total reflectance of multilayer stack}

Differential colorimetry offers a very useful tool for quantifying variations in chromatic interference fringes that arise from spatiotemporal variations in film thickness. The reconstruction method for extracting film shapes eliminates problems often incurred by use of other techniques since human observers perceive colors differently. The technique relies on direct comparison of the RGB (red-green-blue) values of each pixel in a digital image to the predicted RGB colors based on reflectance information from a multilayer stack that includes the deforming liquid film as well the spectral response of the illumination source and the camera used to record the image.$^{15}$ For the system of interest, a multilayer stack was used to compute the (ideal) fraction of incident light reflected from the air/polymer interface as a function of wavelength. Given the relatively large distance between the glass coverslip and the microscope objective, it was assumed that the illumination was normally incident on the planar stack. The ideal or theoretical fractions of RGB for a given film thickness were obtained by convolving the reflection spectrum with the illumination and camera spectral functions. The RGB values from pixels of interest obtained from experimental images were then compared to the theoretical curves and the fit optimized via a cost function.

Given that the liquid peak heights protruding above the initial flat film value $h_{0}$ were typically just a few nanometers, it was difficult to identify the fastest growing modes unambiguously at early stages of the formation process. This problem was resolved by carrying out the peak reconstruction process in inverse chronological order since the peaks of interest were clearly identifiable at late times after they had undergone more growth. A similar approach was successfully implemented in Ref. 11 for the purpose of extracting the wavelength of the fastest growing mode. For each experimental run characterized by $h_{0}, d_{0}$, and $\Delta T$, it was relatively simple to identify the maximum height of the ten fastest growing peaks from image sequences taken at fixed intervals $\Delta t$ in time, where $\Delta t$ ranged from $10 \mathrm{~s}$ to 2 min depending on the control settings governing each run.

The ideal total reflectance was obtained by assuming white light normally incident on a multilayer stack consisting of seven layers arranged in the following sequence-glass, vacuum, sapphire, $\mathrm{SU}-8$, air, polystyrene, and silicon-as indicated in Fig. 2. (For experiments 66 and 69 in Table I in which the SU-8 disk was not used, the stack consisted of only six multilayers.) The values of the refractive index as a function of illumination wavelength $\lambda_{\text {illum }}$ were obtained from the Cauchy equation $^{23}$ given by

$$
n\left(\lambda_{\text {illum }}\right)=B+\frac{C}{\lambda_{\text {illum }}^{2}}+\frac{D}{\lambda_{\text {illum }}^{4}} .
$$

The Cauchy coefficients are listed in Table IV. Silicon has both real and imaginary Cauchy coefficients since the solid absorbs light in the visible part of the spectrum. The refractive index for sapphire was chosen to correspond to the ordinary axis since the orientation of the extraordinary axis in the experiment was unknown. The layer thicknesses in the multilayer stack are listed in Tables I and III. For each material layer, the Cauchy coefficients were substituted into Eq. (7) and the refractive index value $n\left(\lambda_{\text {illum }}\right)$ computed in increments of $1 \mathrm{~nm}$ over the range $0.4 \mu \mathrm{m} \leq \lambda_{\text {illum }} \leq 0.8 \mu \mathrm{m}$. Any unknown interstitial values were obtained by linear interpolation from the two closest values.

Ideal total reflectance values $\mathrm{R}$ for the multilayer stack were computed using the matrix formulation method. ${ }^{29}$ This compact formalism yields a simple $2 \times 2$ matrix description for plane wave reflection, propagation, and transmission through a stack consisting of layers of isotropic homogenous material. The matrix product yields the total transfer matrix, whose elements are used to compute the total reflectance values at normal incidence.

In the analysis below, the planar interfaces are designated by $1 \leq \mathrm{N} \leq 6$ for integer $\mathrm{N}$, and the individual material layers denoted by $0 \leq j \leq N+1$, where the integer $j=1$ represents the glass coverslip layer and $j=6$ represents the silicon wafer. The semi-infinite air layer above the glass coverslip corresponds to

TABLE IV. Cauchy coefficients for evaluating refractive indices as a function of illumination wavelength $\lambda_{\text {illum }}(\mu \mathrm{m})$ within the visible spectrum for the material layers shown in Fig. 2.

\begin{tabular}{lccc}
\hline \hline Material & $\boldsymbol{B}$ & $\begin{array}{c}\boldsymbol{C} \\
\left(\mathbf{1 0}^{-\mathbf{2}} \boldsymbol{\mu \mathbf { m } ^ { \mathbf { 2 } } )}\right.\end{array}$ & $\begin{array}{c}\boldsymbol{D} \\
\left(\mathbf{1 0}^{-\mathbf{4}} \boldsymbol{\mu \mathbf { m } ^ { 4 } )}\right.\end{array}$ \\
\hline Corning 1737 glass (Ref. 24) & 1.505 & 0.455 & -0.218 \\
Vacuum or air (est) & 1.000 & 0.000 & 0.000 \\
Sapphire (ord axis) (Ref. 25) & 1.750 & 0.654 & -1.31 \\
SU-8 (Ref. 26) & 1.566 & 0.796 & 1.40 \\
Polystyrene (Ref. 27) & 1.563 & 0.929 & 1.20 \\
Silicon (real) (Ref. 28) & 3.819 & -17.2 & 727 \\
Silicon (imag) (Ref. 28) & 0.106 & -8.14 & 167 \\
\hline \hline
\end{tabular}


$j=0$. Since there was negligible optical transmission through the silicon wafer, it was assumed that the wafer was bounded below by a semi-infinite air layer denoted by $j=7$. The Fresnel reflection and transmission coefficients for an interface separating layers $j$ and $j+1$ are then given by ${ }^{30}$

$$
\begin{aligned}
r_{j, j+1} & =\frac{n_{j}-n_{j+1}}{n_{j}+n_{j+1}}, \\
t_{j, j+1} & =\frac{2 n_{j}}{n_{j}+n_{j+1}} .
\end{aligned}
$$

The $2 \times 2$ matrix for transmission across an interface separating layer $j$ from layer $j+1$ is given by ${ }^{29}$

$$
\mathbf{T}_{j, j+1}=\frac{1}{t_{j, j+1}}\left[\begin{array}{cc}
1 & r_{j, j+1} \\
r_{j, j+1} & 1
\end{array}\right] .
$$

Similarly, the matrix describing propagation through layer $j$ is given by ${ }^{29}$

$$
\mathbf{T}_{j}=\left[\begin{array}{cc}
e^{-i \delta_{j}} & 0 \\
0 & e^{-i \delta_{j}}
\end{array}\right]
$$

where the phase $\delta_{j}$ obtained when passing through layer $j$ of thickness $z_{j}$ is given by

$$
\delta_{j}\left(\lambda_{\text {illum }}\right)=\frac{2 \pi n_{j}}{\lambda_{\text {illum }}} z_{j} .
$$

The total transfer matrix $\mathbf{M}$ was then computed according to

$$
\mathbf{M}=\left[\begin{array}{ll}
M_{11} & M_{12} \\
M_{21} & M_{22}
\end{array}\right]=\mathbf{T}_{0,1} \mathbf{T}_{1} \mathbf{T}_{1,2} \mathbf{T}_{2} \cdots \mathbf{T}_{\mathrm{N}} \mathbf{T}_{\mathrm{N}, \mathrm{N}+1}
$$

The value of the total reflectance ${ }^{29}$ for pixel location $(\vec{x}, t)$ in a layered stack illuminated by wavelength $\lambda_{\text {illum }}$ for a local film thickness $h(\vec{x}, t)$ was then obtained according to

$$
R\left[\lambda_{\text {illum }}, h(\vec{x}, t)\right]=\frac{\left|M_{21}\right|^{2}}{\left|M_{11}\right|^{2}} .
$$

These values were then inverted to reconstruct the shape and maximum amplitudes of the fastest growing liquid peaks as a function of time.

\section{B. Color maps for extracting instability peak growth rates}

As the linear instability began sprouting protrusions, the recorded image displayed variations in local color due to changes in surface reflectance associated with the changing surface topology of the liquid nanofilm. We sought to quantify the growth rate of the fastest growing modes by examining the RGB content of pixels in the vicinity of emergent liquid peaks. To derive the color cap for an ideal multilayer stack, the tristimulus values for each color channel $\alpha$ were computed from the convolution integral $\mathrm{X}_{\alpha}(h)$, where $\alpha=1 \rightarrow$ red (R), $\alpha=2 \rightarrow$ green $(\mathrm{G})$ and $\alpha=3 \rightarrow$ blue (B) according to

$$
\mathrm{X}_{\alpha}(h)=\int \mathrm{I}\left(\lambda_{\text {illum }}\right) R\left(\lambda_{\text {illum }}, h\right) \mathrm{S}_{\alpha}\left(\lambda_{\text {illum }}\right) d \lambda_{\text {illum }}
$$

where I $\left(\lambda_{\text {illum }}\right)$ denotes the spectral response of the illumination source and $S_{\alpha}\left(\lambda_{\text {illum }}\right)$ denotes the spectral response of the digital camera. Figure 3(a) depicts the normalized function $\mathrm{I}\left(\lambda_{\text {illum }}\right)$ for the halogen source used in this study (Osram HLX $64625,12 \mathrm{~V}, 100 \mathrm{~W})$, which was measured by placing a spectrometer (Ocean Optics USB4000-VIS-NIR) at the focal plane of the nanofilm. The three curves $S_{\alpha}\left(\lambda_{\text {illum }}\right)$ shown in Fig. 3(b) were obtained from the camera manufacturer (DVC 1312C). Shown in Fig. 4(a) are the tristimulus curves computed from Eq. (15) as a function of local film thickness $h$ for the parameter values corresponding to experimental run 56 (see Tables I and III). Each curve was normalized by its maximum value which for the blue channel was outside the range shown. The theoretical RGB map shown in Fig. 4(b) was computed by summing the R, G, and B component values for each value of $h$, in increments of $1 \mathrm{~nm}$. Similar maps were generated for each experimental run.

The color maps were also adjusted to account for automatic changes changes in image brightness and white balance that occurred from one image to another in a given sequence. These changes were enforced through internal camera settings not accessible to users. Image brightness settings, which vary with the exposure time, affected the values of the minima and maxima computed from Eq. (15). The optimal white balance settings set by the camera, internally adjusted by modifying the relative weights of each color channel, also changed from run to run since the actual illumination conditions varied somewhat from experiment to experiment. To account for these effects, we therefore normalized the maximum and minimum values computed from Eq. (15) for each color channel $\alpha$ independently by linearly stretching the curves until the two extrema matched the R, G, and $B$ values extracted from the experiment. This transformation is given by

$$
\begin{aligned}
& \min _{h \in\left[h_{0}, h_{\text {final }}\right]} \widehat{\mathrm{X}}_{\alpha}[h(\vec{x}, t)]=\min _{t \in\left[0, t_{\text {final }}\right]} \mathrm{X}_{\alpha}^{\exp }\left(\vec{x}_{\text {final }}, t\right), \\
& \max _{h \in\left[h_{0}, h_{\text {final }}\right]} \widehat{X}_{\alpha}[h(\vec{x}, t)]=\max _{t \in\left[0, t_{\text {final }}\right]} \mathrm{X}_{\alpha}^{\exp }\left(\vec{x}_{\text {final }}, t\right),
\end{aligned}
$$

where $\vec{x}_{\text {final }}$ denotes the Cartesian coordinate of a pixel analyzed at the final time $t_{\text {final }}$ of an experimental run, $h_{o}$ denotes the local layer thickness at the initial time and $h_{\text {final }}$ denotes the layer thickness corresponding to a local maximum peak height achieved at time $t_{\text {final }}$. For those runs in which the selected protrusions made contact with the cold substrate, the values $h_{\text {final }}$ were set equal to $d_{0}$. However, for those cases in which the gap spacing $d_{0}$ was very large and the liquid did not make contact with the cold substrate (i.e., typically in the absence of the SU-8 disk), the values $h_{\text {final }}$ were estimated by 

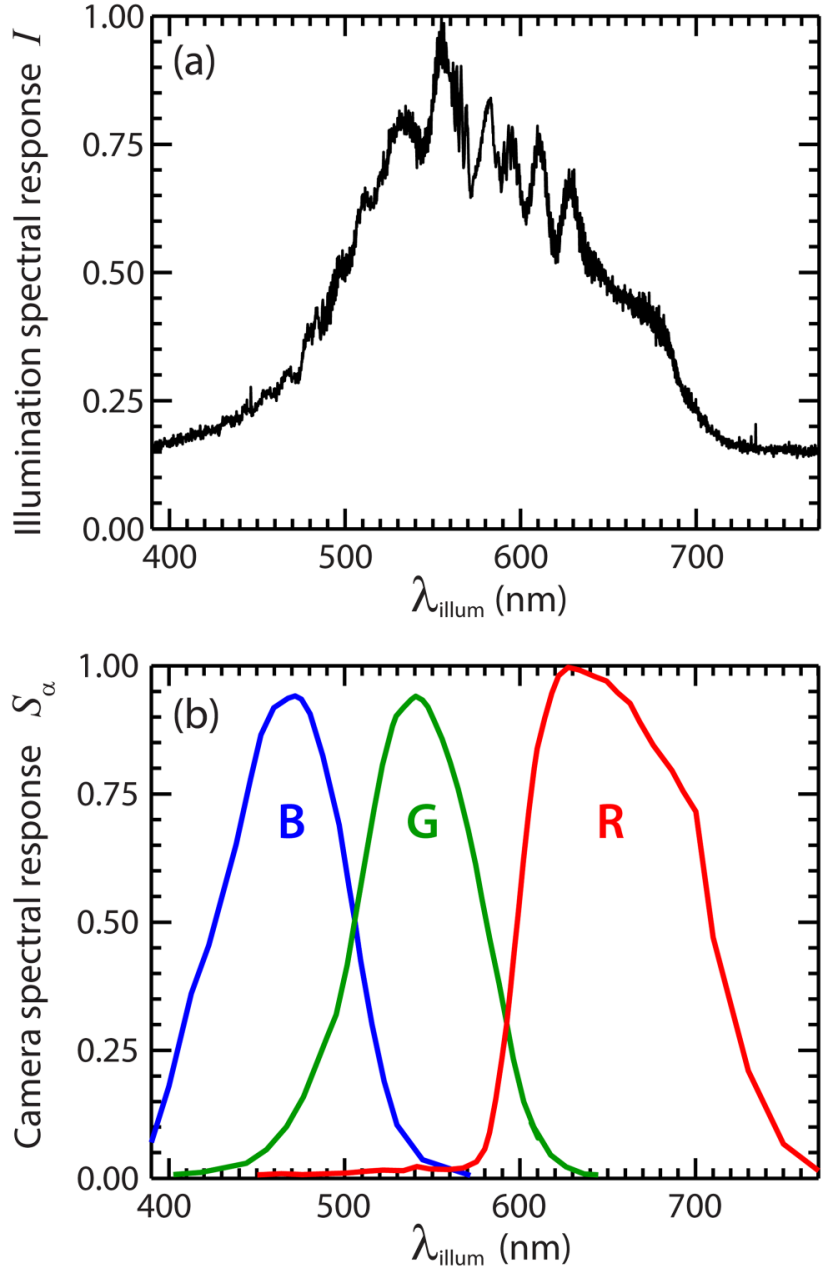

FIG. 3. (a) Measured spectral response $I\left(\lambda_{\text {illum }}\right)$ of halogen light illumination used in this study (Osram HLX 64625, $12 \mathrm{~V}, 100 \mathrm{~W}$ ). (b) Digital camera (DVC $1312 \mathrm{C}$ ) spectral response functions $S_{\alpha}\left(\lambda_{\text {illum }}\right)$ for the individual red $(R)$, green $(G)$, and blue (B) channels as provided by the manufacturer.

matching the experimentally observed color of the selected peaks to the theoretically computed color.

\section{Protocol for the extraction of liquid peak growth rates}

Estimates of the maximum amplitudes $h_{\text {peak }}(t)$ for the ten fastest growing liquid protrusions were obtained as follows. A cost or penalty function $\mathrm{G}(\vec{x}, t, h)$ was defined according to

$$
\mathrm{G}(\vec{x}, t ; h)=\sum_{\alpha=1}^{3}\left\{\mathrm{X}_{\alpha}^{\exp }(\vec{x}, t)-\widehat{\mathrm{X}}_{\alpha}[h(\vec{x}, t)]\right\}^{2}
$$

to minimize the difference between the theoretical and
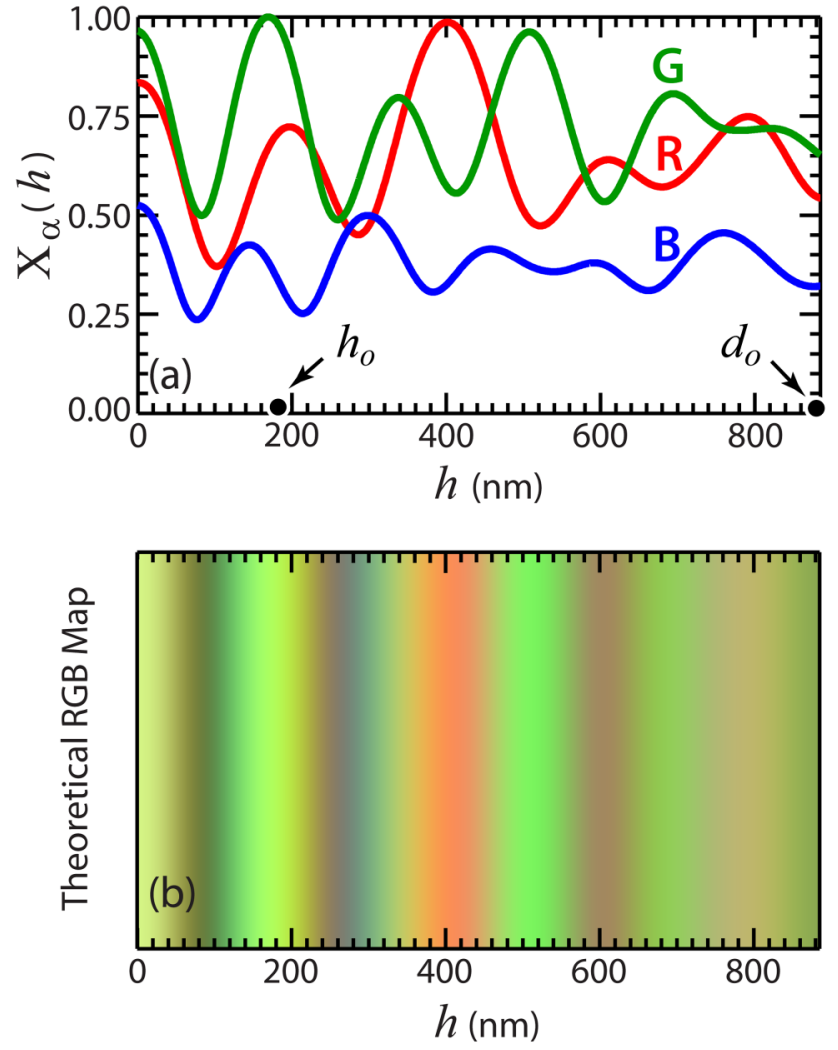

FIG. 4. (a) Tristimulus curves computed according to Eq. (15) as a function of liquid film thickness $h$ for experimental run 56. (Additional input values can be found in Tables I and III.) (b) Theoretical RGB color map computed as described in the text as a function of liquid film thickness $h$.

experimental RGB values. Due to the oscillatory nature of the curves shown in Fig. 4(a) as well as uncertainties in film thickness arising from noisy data or small lateral variations in thickness of the flats comprising the multilayer stack, it was not always possible to find the global minimum of $G(\vec{x}, t, h)$ within the range $h_{0} \leq h \leq h_{\text {final }}$. This was often the case at early times when liquid peak heights had only grown by a few nanometers. For this reason, the image analysis was conducted in reverse chronological order, using the locations and colors of well defined peaks at late times. Starting from a selected peak at the final time, the next numerical search for the peak height explored a neighborhood containing film thicknesses not more than $70 \mathrm{~nm}$ below the peak height value just obtained and so on. Additionally, the algorithm allowed the Cartesian coordinates of the peak being analyzed, i.e., $\left[x_{\text {peak }}(t), y_{\text {peak }}(t)\right]$, to shift between frames by one pixel to the right or left and one pixel up or down. In this way, film elevations for the five pixels within the neighborhood of the peak analyzed in the previous frame were easily computed. This relaxed search scheme accommodated very small lateral shifts in peak locations which occurred from image to image, either due to spurious effects 
or small lateral flow caused by uneven substrates or nearby spacers. The final value accepted, $h_{\text {meas }}(x, y, t)$, was chosen to be the value within the neighborhood of a peak which minimized the cost function $G(\vec{x}, t, h)$ subject to the constraint $h \in\left[h_{\text {peak }}(t+\Delta t)-70 \mathrm{~nm}, h_{\text {peak }}(t+\Delta t)\right]$. The values $h_{\text {peak }}(t)$ and $\left[x_{\text {peak }}(t), y_{\text {peak }}(t)\right]$ so computed identified the location and peak heights at time $t$ for the ten fastest growing peaks.

This procedure is demonstrated next for experiment 56 (see Table I). Four cropped snapshots of the emergent instability are shown in Fig. 5. These images depict the same region of the PS film with initial thickness $h_{0}=183 \mathrm{~nm}$ and $\Delta \mathrm{T}=22.1{ }^{\circ} \mathrm{C}$ at times $\mathrm{t}(\mathrm{min})=4,150,200$ and 400 . Three of the ten fastest growing peaks are circumscribed by white circles. The RGB values of each peak as a function of time were extracted from the peak centroids. Figure 6(a) shows the measured RGB values as a function of time for the peak labeled Top in Fig. 5. The maximum and minimum values of each color curve in Fig. 6(a) were then used to rescale the corresponding theoretical tristimulus curves computed from Eq. (15). Figure 6(b) depicts the resulting match obtained from minimization of the cost function in Eq. (17) between the RGB values extracted experimentally from pixel locations representing peak heights (icons) to those RGB values computed theoretically (solid lines) from the stretched tristimulus curves. Plotting the RGB values for each estimated layer thickness $h$ yielded the color map shown in Fig. 6(c). As evident, the rescaling (i.e., stretching) procedure described above led to a color map which much more closely resembles the colors observed in the experiment-see Fig. 5. In general, this matching procedure worked well for layer thickness values below about $1.5 h_{0}$. Occasionally, it led to small jumps in the reconstructed heights in the vicinity of points, where $\widehat{\mathrm{X}}_{\alpha}[h(\vec{x}, t)]$ reached an extremum. An example of this is evident in Fig. 6(c), near the values $h=500 \mathrm{~nm}$ and $h=750 \mathrm{~nm}$, where both the green and red channels reach an extremum at similar values of $h$. The fitted curves helped bridge the behavior between those discontinuous values in film thickness.

Shown in Fig. 6(d) are the resultant semi-log plots for the reconstructed peak values $h_{\text {peak }}-h_{o}$ for the three protrusions highlighted in Fig. 5. The corresponding growth rates $\sigma_{0}$ were extracted from the slopes of the linear portions of these curves spanning the limits $20 \mathrm{~nm}$ to $h_{0} / 2$. (These two limits were used in all runs.) The linearity observed on the semilog plot confirms exponential growth, in accordance with Eq. (2). However, this behavior typically persisted only for one decade in time or less. In all cases examined, this initial exponential growth gave way to slower than exponential growth at intermediate and late times. This subsequent slower growth was probably caused by two effects, namely, local film depletion surrounding growing protrusions which limited mass flow and increase in viscosity as the liquid peaks approached the cold substrate. The viscosity of low molecular weight polymers $^{31}$ is known to follow Arrhenius-like dependence on temperature, $e^{\Delta E / R T}$, where $\Delta E$ is the activation energy for flow and $\mathrm{R}$ the universal gas constant.

The field of view of images analyzed in this way was fairly large $(1.36 \mathrm{~mm} \times 1.08 \mathrm{~mm})$ and typically encompassed about

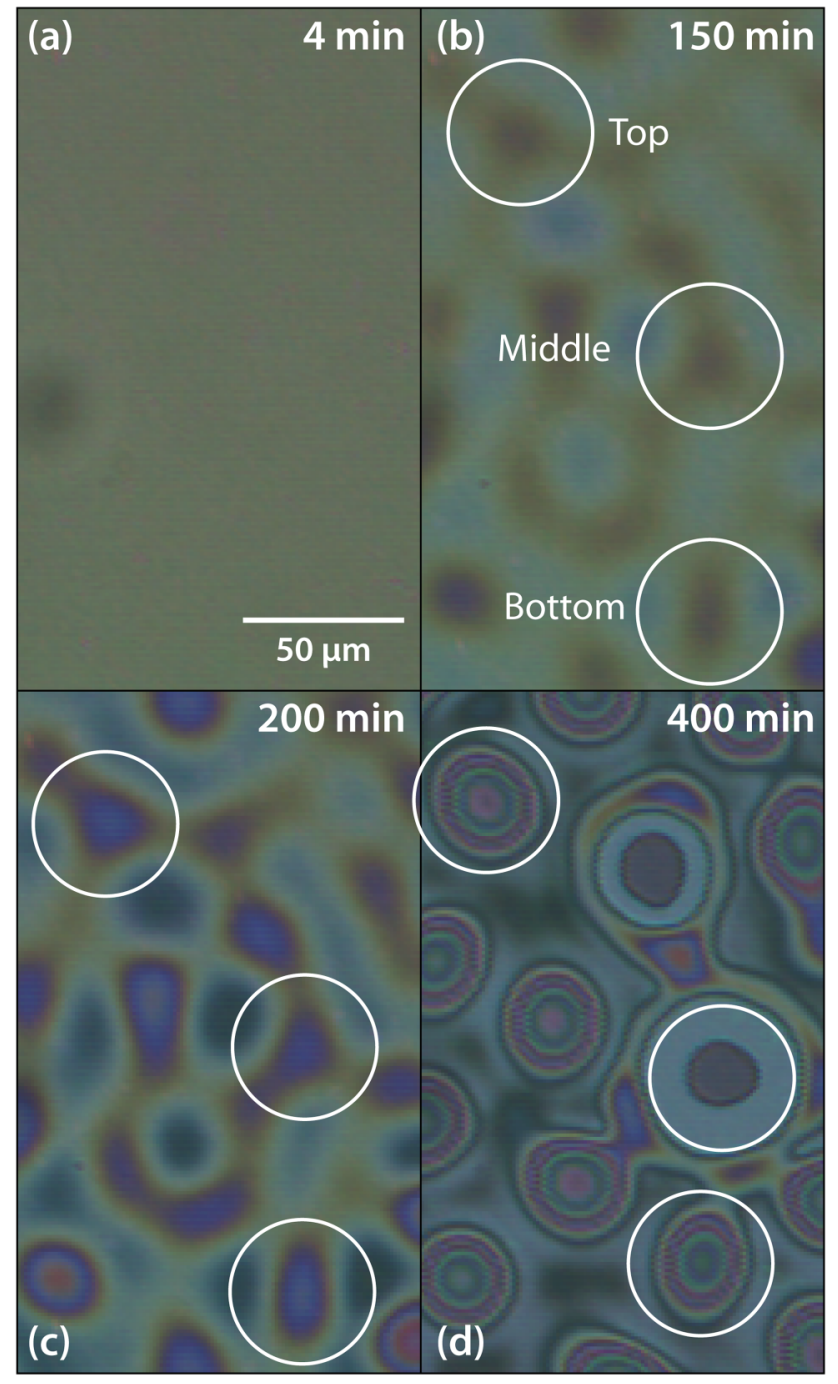

FIG. 5. Four bright field micrographs from experimental run 56 (see Table I) showing the same region of the molten PS film at $t(\min )=4,150,200$, and 400 as it underwent instability. The white circles designate three of the ten fastest growing peaks, labeled top, middle, and bottom for later reference.

100 protrusions. Only the ten fastest growing peaks were selected for analysis. The measured growth rates for the 20 experiments listed in Table I are listed in Table V. While the wave numbers associated with these ten peaks did not necessarily all correspond to the unique wave number $k_{0}$ characterizing the fastest growing mode, we assumed nonetheless that their wavenumbers were closely clustered about that value. This assumption is a reasonable one given that the dispersion curve for the instability is governed by Type-II behavior $^{16}$ and as described by Eq. (3) exhibits a well defined peak about the value $k_{0}$. For each experimental run, we extracted the growth rates $\sigma(k)$ by evaluating the ratio 

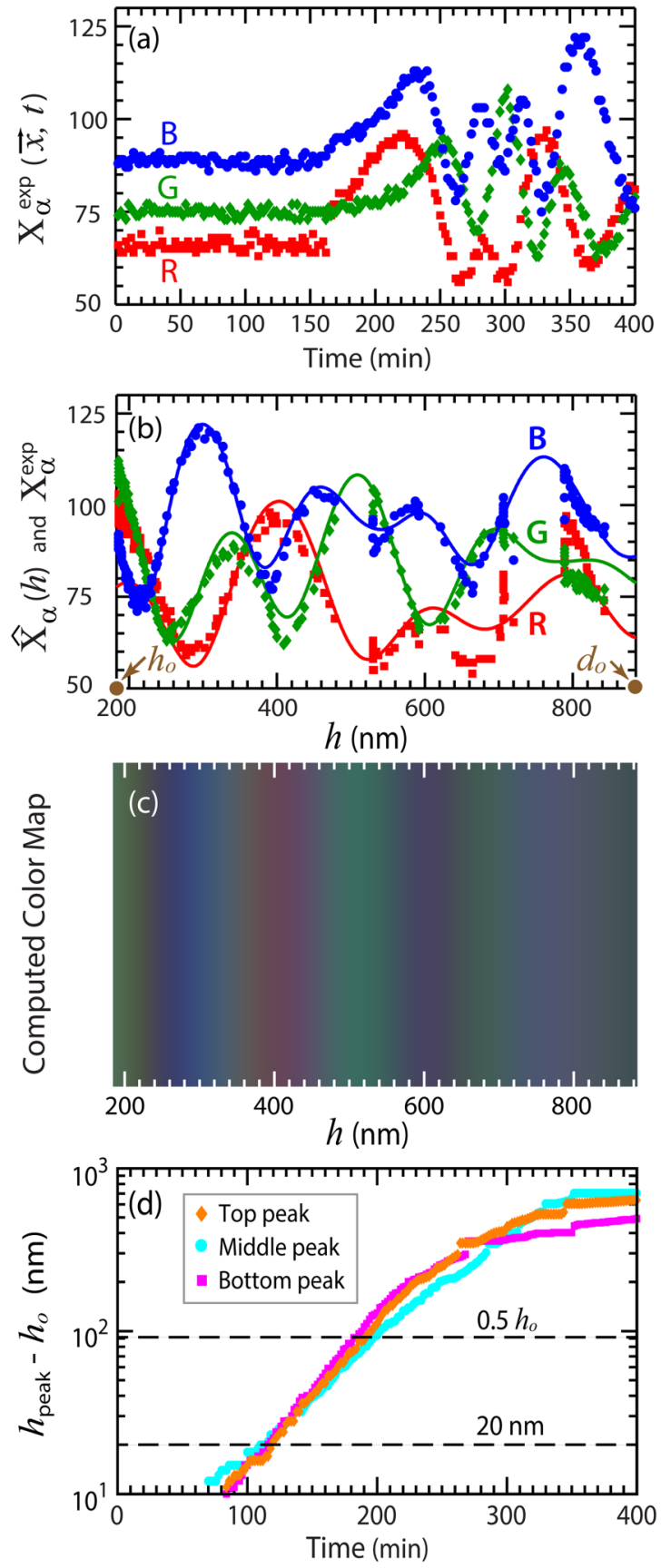

FIG. 6. (a) Measured RGB values as a function of time, $X_{\alpha}^{\exp }(\vec{x}, t)$, for the peak labeled Top in Fig. 5. (b) Match between the tristimulus values extracted from the peak heights in the experiment (icons), $X_{\alpha}^{\exp }(\vec{x}, t)$, with those computed from Eq. (15) and rescaled according to Eq. (16) (solid lines) as obtained from minimization of the cost function in Eq. (17). (c) Color map corresponding to the RGB values given by the solid lines in (b). (d) Reconstruction of the peak height values as a function of time for the three peaks circled in Fig. 5. Linear fits to the curves (not shown) between the limits of $20 \mathrm{~nm}$ and $h_{0} / 2$ were used to extract values of the maximum growth rate $\sigma^{\exp }$. $\ln \left(h_{\text {peak }}-h_{0}\right) / \Delta t$, where $\Delta t$ was the interval in time required for a liquid peak of initial height $20 \mathrm{~nm}$ to attain a height $h_{0} / 2$. We then averaged the growth rates of the ten fastest growing peaks and used that average value $\sigma_{0}^{\text {avg }}$ in reporting the fastest growth rate for each run analyzed. These average values are listed in the last column of Table I.

\section{COMPARISON OF MEASURED GROWTH RATES TO PREDICTIONS FROM THE THERMOCAPILLARY MODEL}

After measuring the growth rates $\sigma_{0}$, we estimated the resulting normalized growth rates given by Eq. (6) using the extrema in PS viscosity given by $\eta\left(\mathrm{T}_{\mathrm{H}}\right)$ and $\eta\left(\mathrm{T}_{\mathrm{C}}\right)$. This served as a check that the data were consistent with early time growth when the film viscosity should closely equal $\eta\left(\mathrm{T}_{\mathrm{H}}\right)$ and not $\eta\left(\mathrm{T}_{\mathrm{C}}\right)$. The viscosities $\eta\left(\mathrm{T}_{\mathrm{H}}\right)$ and $\eta\left(\mathrm{T}_{\mathrm{C}}\right)$ were obtained by linear interpolation in temperature from very low shear measurements on macroscale bulk samples reported by Urakawa et al. ${ }^{18}$ which spanned the range $62^{\circ} \mathrm{C}$ to $216^{\circ} \mathrm{C}$. The polystyrene used in our study was obtained from the same supplier but was of slightly lower molecular weight $\left(M_{w}=1.3 \mathrm{~kg} / \mathrm{mol}\right.$ instead of $\left.M_{w}=1.8 \mathrm{~kg} / \mathrm{mol}\right)$. These limiting values of $\eta$ for each run are listed in Table I. The remaining material constants $-\gamma, d \gamma / d \mathrm{~T}$ and $\kappa$-were held fixed from run to run and estimated according to the values listed in Table II. These values, known to vary slowly with temperature, ${ }^{17}$ were not expected to vary much from run to run given the small range in temperatures applied to the hot substrate $\left(89.8^{\circ} \mathrm{C} \leq \mathrm{T}_{\mathrm{H}} \leq 101^{\circ} \mathrm{C}\right)$.

Shown in Fig. 7 is a plot of the normalized growth rates $\Sigma_{0}=\eta\left(\mathrm{T}_{i}\right) h_{0} \sigma_{0} / \gamma\left(\mathrm{T}_{\mathrm{H}}\right)$, where $i=\mathrm{H}$ or $\mathrm{C}$, given by Eq. (6) using the measured growth rates $\sigma_{0}$ and previously measured values $^{11}$ of the normalized wavelength $\Lambda_{0}=\lambda_{0} / h_{0}$ for the ten fastest growing peaks listed in Table V. Shown for comparison is the relation $\Sigma_{0}=(1 / 3)\left(2 \pi / \Lambda_{0}\right)^{4}$ from Eq. (6) which must be satisfied by any linear instability satisfying a Type II dispersion relation. The experimental data strongly support the predicted scaling $\Lambda_{0}^{-4}$ and show excellent quantitative agreement for estimates based on $\eta(\mathrm{T})=\eta\left(\mathrm{T}_{\mathrm{H}}\right)$.

We also examined the correlation given by Eq. (5) for the growth rate of the fastest growing mode versus normalized separation distance $D=d_{0} / h_{0}$. We note that in recent work, ${ }^{11}$ the best fit between theory and experiment for the fastest growing wavelength $\lambda_{0}=2 \pi / k_{0}$, where $k_{0}$ is given in Eq. (4), was obtained from a least squares fit that yielded the fit constant $C_{\mathrm{TC}}=447\left({ }^{\circ} \mathrm{C}\right)^{1 / 2}$. This fit constant was found to be about $45 \%$ larger than one would estimate from the theoretical relation $C_{\mathrm{TC}}=2 \pi(4 \gamma)^{1 / 2} /(3 \kappa|d \gamma / d \mathrm{~T}|)^{1 / 2}$ using the values of the material constants listed in Table II. For consistency with those earlier measurements of $\lambda_{0}$, we also examined the fit to theory using the same fitting constant to represent the ratio $\mathrm{C}_{\mathrm{TC}}=2 \pi(4 \gamma)^{1 / 2} /(3 \kappa|d \gamma / d \mathrm{~T}|)^{1 / 2}$. To that end, Eq. (5) can be rewritten as

$$
\Sigma_{\mathrm{D}}=\frac{16 \eta\left(\mathrm{T}_{\mathrm{H}}\right) \gamma\left(\mathrm{T}_{\mathrm{H}}\right) h_{o}}{9 \kappa^{2}|d \gamma / d T|^{2}(\Delta \mathrm{T})^{2}} \sigma_{\mathrm{o}}=\frac{\mathrm{D}^{2}}{3(\mathrm{D}+\kappa-1)^{4}},
$$


TABLE V. Measured growth rates $\sigma_{0}\left(10^{-4} / \mathrm{s}\right)$ of the ten fastest growing peaks for each experimental run listed in Table I. Top row designates the number assigned to each experimental run. The ten entries below each run represent the measured growth rates. Within each column, the boldface value below the horizontal line denotes the average (mean) value of the ten measurements, followed by the values of the standard deviation and the relative standard deviation $(\%)$. The minimum $\left(\sigma_{0}^{\min }\right)$, maximum $\left(\sigma_{0}^{\max }\right)$, and average $\left(\sigma_{0}^{\text {avg }}\right)$ values of the growth rate for each experimental run are also reported in the last three columns of Table I.

\begin{tabular}{|c|c|c|c|c|c|c|c|c|c|c|c|c|c|c|c|c|c|c|c|}
\hline 46 & 47 & 48 & 50 & 51 & 52 & 53 & 54 & 56 & 58 & 60 & 61 & 62 & 63 & 64 & 66 & 69 & 71 & 72 & 74 \\
\hline 5.89 & 5.63 & 4.22 & 6.54 & 5.94 & 17.9 & 3.46 & 4.91 & 3.46 & 5.51 & 5.65 & 5.02 & 10.3 & 8.14 & 14.0 & 1.38 & 2.49 & 20.5 & 13.0 & 106 \\
\hline 5.18 & 2.94 & 5.39 & 6.26 & 2.70 & 13.3 & 3.56 & 7.77 & 4.57 & 3.03 & 2.91 & 2.16 & 9.68 & 9.50 & 10.4 & 0.756 & 2.29 & 17.9 & 15.9 & 114 \\
\hline 4.43 & 3.33 & 5.04 & 7.85 & 6.21 & 11.4 & 3.76 & 8.24 & 2.39 & 4.36 & 3.00 & 3.51 & 8.49 & 12.4 & 13.5 & 0.888 & 1.87 & 15.3 & 15.0 & 131 \\
\hline 5.53 & 4.84 & 5.68 & 6.23 & 7.95 & 12.2 & 2.87 & 7.08 & 3.06 & 3.38 & 5.17 & 4.45 & 8.45 & 5.56 & 23.8 & 1.24 & 2.09 & 17.8 & 12.3 & 131 \\
\hline 5.65 & 2.99 & 4.50 & 8.13 & 8.69 & 15.1 & 3.94 & 10.7 & 3.76 & 3.00 & 3.45 & 4.38 & 12.1 & 6.31 & 17.2 & 1.07 & 1.62 & 13.4 & 13.9 & 84.5 \\
\hline 5.03 & 3.69 & 4.53 & 4.99 & 7.84 & 13.2 & 3.75 & 5.86 & 3.39 & 4.05 & 3.03 & 4.74 & 10.5 & 7.59 & 17.9 & 0.962 & 1.63 & 14.1 & 15.5 & 151 \\
\hline 4.48 & 7.79 & 4.79 & 6.29 & 7.56 & 12.2 & 4.73 & 9.75 & 4.68 & 3.82 & 3.88 & 5.28 & 10.8 & 10.6 & 16.1 & 0.848 & 2.03 & 21.0 & 11.8 & 112 \\
\hline 4.11 & 2.99 & 5.46 & 6.89 & 6.52 & 14.4 & 3.45 & 9.96 & 2.96 & 1.83 & 3.42 & 3.63 & 10.2 & 12.8 & 11.9 & 1.25 & 1.85 & 21.9 & 13.7 & 136 \\
\hline 5.03 & 4.43 & 4.39 & 5.45 & 8.74 & 12.3 & 5.23 & 4.73 & 3.73 & 2.96 & 3.14 & 4.86 & 7.03 & 5.09 & 13.6 & 0.345 & 2.03 & 12.1 & 10.9 & 158 \\
\hline 4.97 & 3.19 & 3.84 & 7.17 & 7.94 & 9.45 & 3.15 & 6.35 & 2.95 & 4.07 & 4.23 & 6.10 & 10.8 & 8.55 & 12.2 & 1.00 & 2.03 & 22.5 & 12.9 & 99.0 \\
\hline 5.03 & 4.18 & 4.78 & 6.58 & 7.01 & 13.1 & 3.79 & 7.54 & 3.50 & 3.60 & 3.79 & 4.41 & 9.84 & 8.65 & 15.1 & 0.973 & 1.99 & 17.7 & 13.5 & 122 \\
\hline 0.569 & 1.56 & 0.596 & 0.976 & 1.80 & 2.29 & 0.708 & 2.12 & 0.722 & 0.999 & 0.955 & 1.09 & 1.47 & 2.69 & 3.88 & 0.296 & 0.27 & 3.77 & 1.63 & 23.2 \\
\hline 11.3 & 37.4 & 12.5 & 14.8 & 25.7 & 17.4 & 18.7 & 28.2 & 20.7 & 27.7 & 25.2 & 24.8 & 14.9 & 31.0 & 25.8 & 30.4 & 13.5 & 21.4 & 12.1 & 19.0 \\
\hline
\end{tabular}

which is then re-expressed in terms of $\mathrm{C}_{\mathrm{TC}}$, according to which

$$
\Sigma_{\mathrm{D}}=\frac{\eta\left(\mathrm{T}_{\mathrm{H}}\right) h_{o}\left(\mathrm{C}_{\mathrm{TC}}\right)^{4}}{(2 \pi)^{4} \gamma\left(\mathrm{T}_{\mathrm{H}}\right)(\Delta \mathrm{T})^{2}} \sigma_{\mathrm{o}}=\frac{\mathrm{D}^{2}}{3(\mathrm{D}+\kappa-1)^{4}} .
$$

Plotted in Fig. 8 are the measured values of $\Sigma_{\mathrm{D}}$ estimated from Eq. (18), where $C_{\mathrm{TC}}=2 \pi(4 \gamma)^{1 / 2} /(3 \kappa|d \gamma / d \mathrm{~T}|)^{1 / 2}$ and from Eq. (19) with $C_{\mathrm{TC}}=447\left({ }^{\circ} \mathrm{C}\right)^{1 / 2}$. Included for comparison is the curve $D^{2} / 3(D+\kappa-1)^{4}$. Clearly, the relation incorporating the fit constant $\mathrm{C}_{\mathrm{TC}}=447\left({ }^{\circ} \mathrm{C}\right)^{1 / 2}$ yields better quantitative agreement with the growth rates predicted by linear stability analysis.

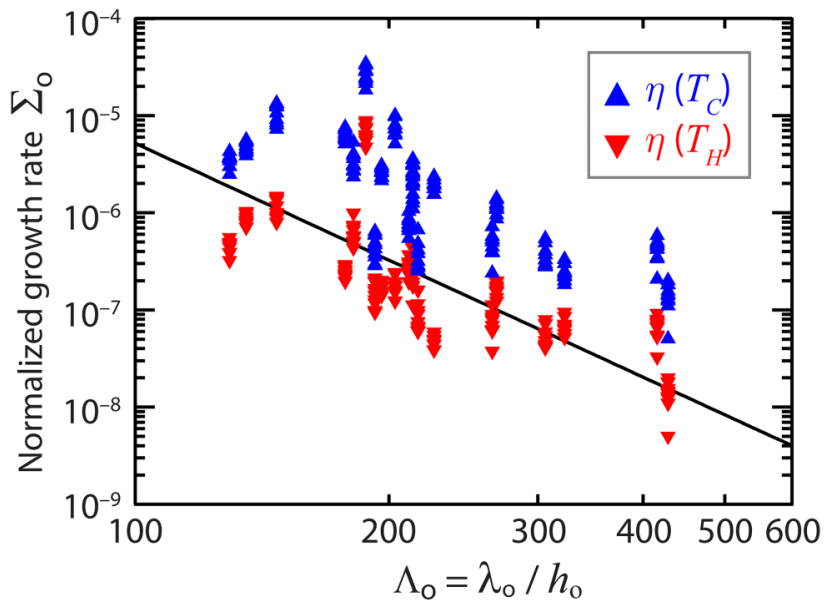

FIG. 7. Correlation between the normalized growth rate and normalized wavelength of the fastest growing mode. Triangles (blue): Normalized growth rates $\eta\left(T_{H}\right) h_{0} \sigma_{0} / \gamma\left(T_{H}\right)$. Inverted triangles (red): Normalized growth rates $\eta\left(T_{C}\right) h_{0} \sigma_{0} / \gamma\left(T_{H}\right)$. Solid line (black): Eq. (6).

\section{DISCUSSION}

Experimental studies designed to measure the growth rates of liquid protrusions stemming from the thermal instability described clearly stand to benefit from the collection of more data in order to reduce the scatter observed in Figs. 7 and 8. Despite the many precautions taken in our study, the experiments proved quite challenging nonetheless for a number of reasons which ultimately generated the scatter observed.

\section{A. Quality of nanofilms}

Larger data sets to help reduce data scatter will ultimately require fabrication of higher quality nanofilms with

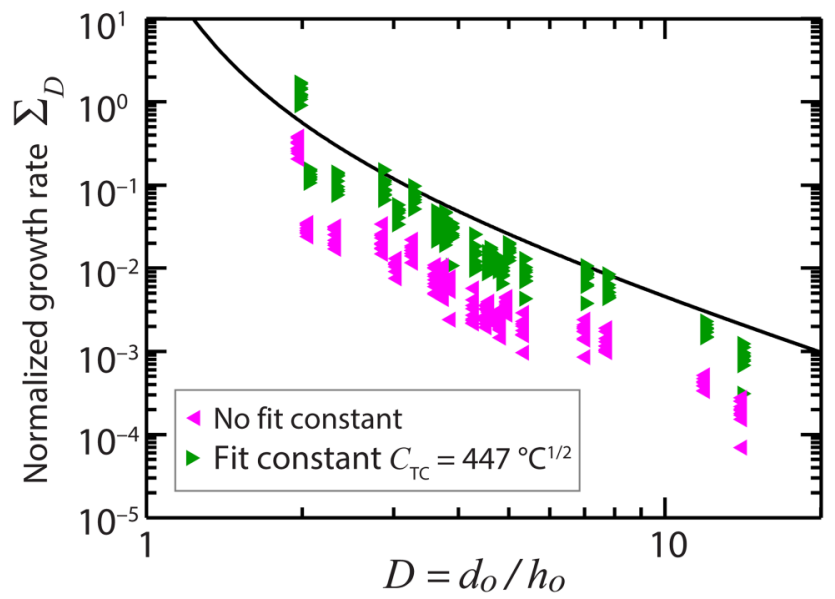

FIG. 8. Normalized instability growth rates $\Sigma_{D}$ versus normalized separation distance $D=d_{0} / h_{0}$ given by Eqs. (18) and (19). The solid curve represents the function $D^{2} / 3(D+\kappa-1)^{4}$. 
few, if any, defects in order to provide larger areas of the film for optical analysis. Despite having tried various cleaning and film preparation techniques over the years, we continue to observe that even seemingly uniform and homogeneous solid polymer nanofilms, once heated, undergo nucleation and growth of pinholes which subsequently trigger polymer dewetting fronts that interfere with the development and measurement of the intrinsic thermocapillary instability. Some researchers ${ }^{32}$ have reported that pinholes in heated nanofilms are unavoidable since they may originate from a wetting transition in nanofilms triggered by heating. While at cooler temperatures the van der Waals interactions between the supporting silicon substrate and the polystyrene nanofilm is attractive (wetting), warmer temperatures can induce repulsion (dewetting). Other studies ${ }^{33}$ have reported that the presence of even a small amount of residual solvent (e.g., toluene) in spun coat nanofilms can ultimately lead to the formation of localized pockets that upon solvent evaporation also trigger pinhole formation. In addition to such intrinsic problems leading to film defects, there is also the issue of contamination from external sources. To mitigate this problem, we positioned our optical apparatus in front of a large horizontal laminar flow clean bench (Clean Air Products, Series 303) placed inside a large enclosure encircled by flexible lab curtains to clear contaminants away from the optical microscope. Future studies would likely benefit significantly from full operation in a Class 1000 or better clean room including film preparation, deposition, and optical measurements.

\section{B. Reproducibility}

Ideally, we would have liked to develop a reliable protocol whereby each polymer sample of a given film thickness $h_{o}$ could be subjected to the thermal instability several times but subject to different values of $d_{o}$ and $\Delta \mathrm{T}$, which would surely have improved the data quality. To accomplish this, we anticipated that after a run was concluded, the instability could be terminated by raising the temperature of the cold substrate to $\mathrm{T}_{\mathrm{H}}$, thereby eliminating the thermal gradient and only relying on capillary forces to level the film back to its initial state. Despite several attempts at this, the protocol failed to work successfully in practice for reasons not evident. The resulting film was never as flat and uniform as the origin spun coat film. Furthermore with both substrates held at $\mathrm{T}_{\mathrm{H}}$, the PS film near the spacers tended to dewet from the posts, which then triggered rapid dewetting fronts that expelled PS film from the measurement area defined. Therefore, each new run required that a new coated substrate be inserted into the apparatus shown in Fig. 2 and re-leveled by hand using the screw assembly described earlier. Depending on the area of the film which exhibited the best quality (i.e., few or no pinholes), the actual value of $d_{0}$ in the vicinity may not have exactly corresponded to the value of $d_{o}$ obtained by the profilometric measurements of the spacers made ex situ.

\section{Sources of uncertainty}

Errors were likely therefore incurred in estimating the actual normalized distances $D=d_{o} / h_{o}$ separating the hot and cold substrates confining the polymer melt. According to Eqs. (18) and (19), the normalized growth rates scale as $\mathrm{D}^{-2}$ and therefore errors in $d_{o}$ and $h_{o}$ exacerbate errors in estimates of D. Our reported values of D are based on ex situ measurements of $d_{o}$ and $h_{0}$ described in Sec. III. During the course of each experiment, however, we noticed two features that likely led to operational uncertainties in estimating D. First, the vertical screws used to enforce parallelism between the aluminum and sapphire substrates sometimes caused the cylindrical posts used as spacers to bend or shift slightly when in contact with the molten film. Tightening of the screws also tended to squeeze the polymer film immediately beneath these cylindrical posts leading to nonuniformities in $h_{0}$ and $d_{0}$. Both these effects, too challenging to quantify in any meaningful way with our current setup, likely contributed to the data scatter evident in Figs. 7 and 8. Also as previously described, direct measurement of $\Delta \mathrm{T}$ was not possible and estimated values had to be obtained instead from finite element simulations, ${ }^{11}$ which relied on thermal conductivity values obtained from the literature (and not necessarily at exactly the operating temperatures used in our study) for all the material layers shown in Fig. 2.

Aside from these uncertainties in $d_{0}, h_{0}$, and $\Delta \mathrm{T}$, another possible source of error may stem from lack of knowledge of the true values of the four material constants required to make contact with the theoretical model, namely, the polymer melt viscosity $\eta$, surface tension $\gamma$, thermocapillary coefficient $\partial \gamma / \partial \mathrm{T}$, and thermal conductivity ratio $\kappa$. As discussed earlier, the values we used in this study were extracted from the literature and represent values obtained with bulk samples and at temperatures as close as we could find to our actual operating temperatures, which was not always possible. There is also the possibility that nanofilms prepared by spin coating manifest a molecular structure different than in bulk because of influence established by the centrifugation process which might have induced orientational order further enhanced by rapid evaporation of toluene. Unfortunately, there are currently no known reliable techniques for obtaining direct measurements of these constants in ultrathin liquid films so it is currently impossible to estimate the errors introduced in our analysis due to uncertainty in these material properties.

The model to which the data were compared assumes that the unstable film does not undergo any phase change such as solidification during growth. All researchers to date who have investigated this thermally induced instability have used low molecular weight polymer melts. The reason for this is the higher viscosities, in comparison to non-polymeric films, slow the development and growth of protrusions, facilitating lengthier observation times for improved measurement. This use, however, raises question as to whether such polymeric films can undergo any phase change or perhaps manifest non-Newtonian behavior during the course of measurement. In our study, the PS films, whose glass transition 
temperature was estimated to be about $\mathrm{T}_{g}=60^{\circ} \mathrm{C}$, were subject to hot temperatures $89.9{ }^{\circ} \mathrm{C} \leq \mathrm{T}_{\mathrm{H}} \leq 101^{\circ} \mathrm{C}$ and cold temperatures $67.8^{\circ} \mathrm{C} \leq \mathrm{T}_{\mathrm{C}} \leq 88.7^{\circ} \mathrm{C}$. As a result, no phase change occurred even when the liquid protrusions approached the cold substrate, though all the measurements reported were terminated when the growth amplitude $h_{\text {peak }}-h_{0}$ attained a value $h_{0} / 2$. No phase change therefore occurred.

We also estimated the maximum shear rate induced by thermocapillary forces at the air/liquid interface to determine whether non-Newtonian behavior might be expected. The characteristic shear rate, relevant to early time deformation of the film, was estimated from the ratio $u_{c} / h_{o}$, where $u_{c}$ is the characteristic thermocapillary flow speed given by Eq. (21) of Ref. 9, namely,

$$
u_{\mathrm{c}}=\frac{h_{\mathrm{o}} \kappa \mathrm{D}|d \gamma / d \mathrm{~T}| \Delta \mathrm{T}}{\eta(\mathrm{D}+\kappa-1)^{2}} .
$$

Substituting values from experiments conducted either at the maximum value of $\Delta \mathrm{T}$ or yielding the smallest value of $\lambda_{\mathrm{o}}$ indicated very low shear rates in the range of $1.0-1.64 \mathrm{~s}^{-1}$, thereby eliminating concern over possible non-Newtonian behavior.

\section{CONCLUSION}

In this work, we implemented a differential colorimetry technique to quantify the growth rate of fluctuations in liquid nanofilms and compared these measurements to model predictions ${ }^{8,9}$ which attribute these formations to a long wavelength thermocapillary instability. At early times, the spacing and growth rate of fluctuations in film thickness are controlled by the competition between large destabilizing thermocapillary stresses and large stabilizing capillary stresses. Linear stability analysis offers an explicit dispersion relation for the rate of growth of the fastest growing unstable mode as a function of geometric parameters and material constants. The measurements reported here were somewhat challenging to carry out since the fluctuation amplitudes within the linear regime spanned only about $20-200 \mathrm{~nm}$. The peak reconstruction process could therefore not be obtained by white light or laser interferometry. Instead, extraction of the growth rate of liquid peak heights relied on reflectance measurements from the air/liquid interface as it deformed in time. The peak reconstruction process incorporated numerous factors affecting the overall reflectance including the spectral response of the camera and illumination source, variations between images due to camera brightness and white balance settings, and small lateral drifts in peak positions caused by slightly non-parallel substrates or other artifacts.

Despite the shortcomings described in conducting this study, the measured growth rates show good quantitative agreement with the predictions of the thermocapillary model. This agreement lends further support to the hypothesis that fluctuation growth in such systems is triggered by a long wavelength instability in which thermocapillary stresses are sufficiently strong to pull fluid elongations out-of plane toward the colder proximate substrate. While this study helps further establish the correlation between the instability growth rate and the normalized wavelength of the fastest growing mode $\Lambda_{0}=\lambda_{0} / h_{0}$ and normalized gap ratio $D=d_{0} / h_{0}$, we were unable to test the correlation with $\Delta \mathrm{T}$ since the experimental setup could only generate values $10.6^{\circ} \mathrm{C} \leq \Delta \mathrm{T} \leq 25.8^{\circ} \mathrm{C}$. Efforts are underway to redesign the system in the hopes of achieving at least one full decade increase in $\Delta \mathrm{T}$, if not more.

\section{ACKNOWLEDGMENTS}

The authors acknowledge the financial support from the National Science Foundation (NSF) (No. CBET 0701324) and a 2013 NASA Space Technology Research Fellowship to K. R. Fiedler (No. NNX13AN41H). We are also grateful for the use of instrumentation provided by the Molecular Materials Research Center of the Beckman Institute of the California Institute of Technology.

\section{REFERENCES}

${ }^{1}$ S. Van Hook, M. F. Schatz, W. D. McCormick, J. B. Swift, and H. L. Swinney, Phys. Rev. Lett. 75, 4397 (1995).

${ }^{2}$ S. Van Hook, M. F. Schatz, J. B. Swift, W. D. McCormick, and H. L. Swinney, J. Fluid Mech. 345, 45 (1997).

${ }^{3}$ N. Garnier, R. O. Grigoriev, and M. F. Schatz, Phys. Rev. Lett. 91, 054501 (2003).

${ }^{4}$ S. Y. Chou and L. Zhuang, J. Vac. Sci. Technol. B 17, 3197 (1999).

${ }^{5}$ L. Zhuang, "Controlled self-assembly in homopolymer and diblock copolymer," Ph.D. thesis (Princeton University, Princeton, NJ, 2002).

${ }^{6}$ E. Schäffer, T. Thurn-Albrecht, T. P. Russell, and U. Steiner, Nature 403, 874 (2000).

${ }^{7_{K}}$. A. Leach, Z. Lin, and T. P. Russell, Macromolecules 38, 4868 (2005).

${ }^{8}$ M. Dietzel and S. M. Troian, Phys. Rev. Lett. 103, 074501 (2009)

${ }^{9}$ M. Dietzel and S. M. Troian, J. Appl. Phys. 108, 074308 (2010).

${ }^{10}$ E. McLeod, Y. Liu, and S. M. Troian, Phys. Rev. Lett. 106, 175501 (2011).

${ }^{11}$ K. R. Fiedler and S. M. Troian, J. Appl. Phys. 120, 205303 (2016).

${ }^{12}$ T. P. Russell and J. Bae, in Polymers, Liquids and Colloids in Electric Fields: Interfacial Instabilities, Orientation and Phase Transitions, Series in Soft Condensed Matter, edited by Y. Tsori and U. Steiner (World Scientific Publishing Co., 2009), Vol. 2, pp. 113-147.

${ }^{13}$ E. Schäffer, T. Thurn-Albrecht, T. P. Russell, and U. Steiner, Europhys. Lett. 53, 518 (2001).

${ }^{14}$ K. A. Leach, S. Gupta, M. D. Dickey, C. G. Willson, and T. P. Russell, Chaos 15, 047506 (2005).

${ }^{15}$ M. Hartl, I. Krupka, and M. Liska, Opt. Eng. 36, 2384 (1997).

${ }^{16} \mathrm{M}$. Cross and H. Greenside, Pattern Formation and Dynamics in Nonequilibrium Systems (Cambridge University Press, 2009).

${ }^{17}$ Z. Pu, in Polymer Data Handbook, edited by J. E. Mark (Oxford University Press, New York, 1999).

${ }^{18}$ O. Urakawa, S. F. Swallen, M. D. Ediger, and E. D. von Meerwall, Macromolecules 37, 1558 (2004).

${ }^{19}$ J. R. Welty, C. E. Wicks, and R. E. Wilson, Fundamentals of Momentum, Heat, and Mass Transfer, 3rd ed. (John Wiley and Sons, 1984).

${ }^{20}$ E. Schäffer, S. Harkema, M. Roerdink, R. Blossey, and U. Steiner, Adv. Mater. 15, 514 (2003).

${ }^{21}$ E. Schäffer, S. Harkema, R. Blossey, and U. Steiner, Europhys. Lett. 60, 255 (2002)

${ }^{\mathbf{2 2}}$ E. Schäffer, "Instabilities in thin polymer films: Structure formation and pattern transfer," Ph.D. thesis (Konstanz University, 2001).

${ }^{23}$ F. A. Jenkins and H. E. White, Fundamentals of Optics, 4th ed. (McGraw-Hill Inc., 1976).

${ }^{\mathbf{2 4}}$ Corning, Inc., Corning Eagle $\mathrm{XG}^{\circledR}$, Corning, NY.

${ }^{25}$ Tydex Optics, Tydex Sapphire ${ }^{\circledR}$, St. Petersburg, Russia.

${ }^{26}$ MicroChem Corp., SU-8 $2000^{\circledR}$, Westborough, MA. 
27I. D. Nikolov and C. D. Ivanov, Appl. Optics 39, 2067 (2000).

${ }^{28}$ M. A. Green and M. J. Keevers, Prog. Photovolt. 3, 189 (1995).

${ }^{29} \mathrm{P}$. Yeh, Optical Waves in Layered Media (John Wiley and Sons, Inc., Hoboken, NJ, 2005).

${ }^{30} \mathrm{M}$. Born and E. Wolf, Principles of Optics: Electromagnetic Theory of Propagation, Interference and Diffraction of Light (Cambridge University Press, 1999).
${ }^{31}$ R. B. Bird, R. C. Armstrong, and O. Hassager, Dynamics of Polymeric Liquids: Vol. 1 Fluid Mechanics, 2nd ed. (John Wiley \& Sons, Inc., New York, 1987).

${ }^{32}$ K. M. Ashley, D. Raghavan, F. Douglas, and A. Karim, Langmuir 21, 9518 (2005).

${ }^{33}$ S. H. Lee, P. J. Yoo, S. J. Kwon, and H. H. Lee, J. Chem. Phys. 121, 4346 (2004). 